\title{
LETTERS
}

\section{Failing a re-treatment regimen does not predict MDR/ XDR tuberculosis: is "blind" treatment dangerous?}

\section{To the Editors:}

Multidrug-resistant (MDR) and extensively drug-resistant (XDR) tuberculosis (TB) represent an emerging public health threat worldwide [1]. The Stop TB Strategy has been revised to provide universal access to diagnosis and treatment for all patients with MDR-TB by 2015 [2-4]. This plan calls for accelerated access to culture for mycobacteria, species identification and drug susceptibility testing (DST) of Mycobacterium tuberculosis. However, in several countries, appropriate TB laboratory facilities are still largely unavailable. In the absence of culture and DST, TB patients failing a re-treatment regimen have been considered eligible for treatment regimens with second-line drugs, under the assumption that $\geqslant 80 \%$ of them would harbour MDR M. tuberculosis strains [5].

In Burkina Faso, as in most low- and some middle-income settings, pulmonary TB cases failing a standard category II regimen are only identified by positive sputum smear microscopy at the end of the fifth month of treatment and are defined as "chronic" TB patients. The diagnosis of chronic TB has significant implications. National guidelines for the management of chronic TB cases in Burkina Faso recommend hospital admission and in-patient treatment for $\geqslant 6$ months with a standardised category IV treatment regimen [6].

Although the diagnosis of chronic TB is taken as a surrogate marker for the identification of MDR-TB, little evidence is available from the literature on its positive predictive value for MDR-TB diagnosis. We systematically performed microbiological investigations, which included both phenotypic and genotypic techniques, on all newly identified chronic TB cases diagnosed over a 2-yr period in Burkina Faso. Here, we report the results and discuss the implications that "blind" treatment with second-line drugs have at programmatic level.

A prospective, country-wide investigation was performed to microbiologically characterise all consecutively registered chronic TB cases in Burkina Faso, defined on the basis of positive sputum smear microscopy at the fifth month of a category II standard re-treatment course (2 months of isoniazid $(\mathrm{H})$, rifampicin $(\mathrm{R})$, pyrazinamide $(\mathrm{Z})$, ethambutol $(\mathrm{E})$ and streptocomycin (S) (2HRZES) then 1HRZE, followed by 5HRE). Cases were recruited at the two national reference centres for chronic TB treatment (the Pneumophysiology Services at the University Hospitals of Ouagadougou and Bobo Dioulasso) from January 2007 to December 2008.

According to national guidelines, registered chronic TB patients were started on a standard, Green Light Project-approved, category IV regimen, which consisted of an intensive phase of 6 months with pyrazinamide, ofloxacin, kanamycin, ethionamide and cycloserine, followed by a 15-month continuation phase with ofloxacin, ethionamide and cycloserine.

Sputum samples were collected prior to starting treatment or within 4 months from its start. Two early morning sputum samples were collected and immediately frozen at $-20^{\circ} \mathrm{C}$ in Ouagadougou before being shipped to Milan, Italy in dry ice packaging. Samples were then decontaminated and processed for smear microscopy. Culture and first-line DST on all $M$. tuberculosis isolates were performed using a BACTEC ${ }^{\mathrm{TM}}$ MGIT960 ${ }^{\mathrm{TM}}$ Mycobacterial Detection System (Becton Dickinson, Cockeysville, MD, USA) according to the manufacturer's instructions.

Second-line DST (ofloxacin, kanamycin, amikacin, capreomycin, ethionamide and cycloserine) was performed on all MDR-TB isolates using an MGIT960 according to published standards [7]. Decontaminated specimens were also analysed for the presence of mutations conferring resistance to rifampicin and isoniazid by the molecular assay GenoType MTBDRplus (Hain Lifescience, Nehren, Germany) as described previously [8].

Out of 83 chronic TB cases registered, 63 (75.9\%) cases provided sputum samples for culture and PCR examination, and were enrolled in the study (fig. 1). Samples were collected from patients before the beginning of treatment in 57 patients, whereas three, two and one patient(s) were sampled at months 2, 3 and 4 after starting therapy, respectively.

A test for antibodies against HIV was accepted by all the 63 patients and was positive in $12(19 \%)$ patients.

M. tuberculosis-positive culture was obtained in 38 (60.3\%) cases, whereas $13(20.6 \%)$ cases harboured nontuberculous mycobacteria (NTM). 12 (19.1\%) samples were culture-negative. Among the six cases sampled after starting second-line TB regimens, four harboured a M. tuberculosis strain and one an NTM; one case resulted culture-negative.

Of the $38 \mathrm{M}$. tuberculosis culture-positive samples, 25 (65.8\%) samples were MDR at first-line DST. Seven were resistant to isoniazid, and six were susceptible to both rifampicin and isoniazid. The positive predictive value of the diagnosis of chronic TB for MDR-TB was 39.7\% (25 MDR cases out of 63 chronic TB patients tested).

No XDR strains were identified by second-line DST: only two strains resulted resistant to amikacin, while all 25 were fully susceptible to quinolones.

The GenoType MTBDRplus assay detected M. tuberculosis complex DNA in all the $38 \mathrm{M}$. tuberculosis culture-positive cases. All but two MDR-TB cases were confirmed by GenoType 


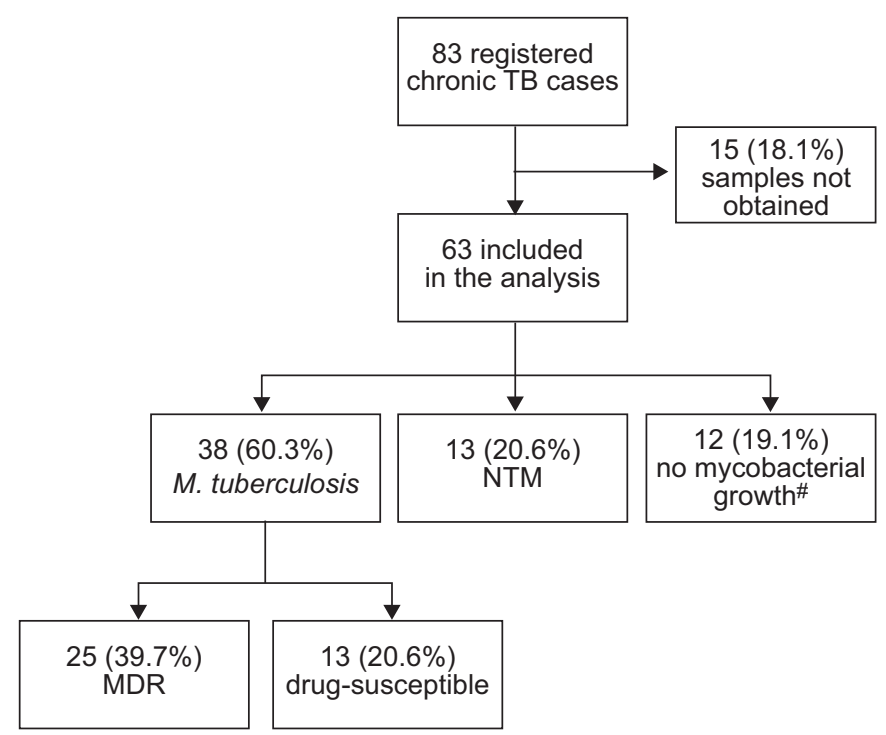

FIGURE 1. Flow chart of chronic tuberculosis (TB) patients and related strains in Burkina Faso, 2007-2008. NTM: nontuberculous mycobacteria; MDR: multidrugresistant. "* nine out of 12 cases positive for Mycobacterium tuberculosis DNA, sensitive to rifampin and isoniazid.

MTBDRplus assay performed on samples and confirmed on isolated strains. Of the two discrepant cases, one was phenotypically sensitive to rifampicin (with a borderline minimum inhibitory concentration of $2 \mu \mathrm{g} \cdot \mathrm{mL}^{-1}$ ) and harboured mutations (M515I and H526N) in the rpoB gene, and one was phenotypically resistant to isoniazid but did not show any of the mutations included in the GenoType MTBDRplus assay. Of the 13 NTM culture-positive patients, infections due to $M$. avium (five cases), M. intracellulare (four cases), M. simiae (three cases), and M. chelonae (one case) were observed. Four patients were HIV-coinfected.

PCR investigations on the 13 cases harbouring NTMs resulted negative for $M$. tuberculosis complex DNA in 11 patients; NTMs were isolated in two $M$. tuberculosis complex DNA-positive cases susceptible to rifampicin and isoniazid.

The PCR tests on the 12 culture-negative cases resulted negative for mycobacterial DNA in three cases, while in nine cases, M. tuberculosis genomic DNA was detected, but mutations conferring rifampicin or isoniazid resistance were not detected.

We have shown that patients failing a re-treatment regimen (currently defined as chronic TB cases) have a probability of only $40 \%$ to represent MDR-TB cases. Conversely, almost twothirds of the cases managed as chronic under programme conditions in Burkina Faso were not MDR-TB, harbouring drug-susceptible or NTM strains instead. The misclassification of chronic TB cases has several implications. First, it elicits unnecessary use of second-line drugs, which are considerably more toxic and expensive than first-line ones and require longer treatment duration. Secondly, in Burkina Faso and other settings in Africa, Asia and Eastern Europe (the former Soviet Union, in particular), chronic TB patients are hospitalised for $\geqslant 6$ months in facilities admitting other patients at high risk for contracting MDR-TB; in the presence of suboptimal infection control measures, the risk of nosocomial transmission of MDRTB is significant [9]. Thirdly, the blind management of twothirds of the cases is likely to foster the development of further resistance and favour the emergence of XDR-TB strains [10, 11].

Sputum smear microscopy remains a mainstay for TB diagnosis, but it is clearly inadequate for the management of patients failing a re-treatment regimen. Whether it is also inadequate for the management of patients failing a category I regimen should soon be investigated. The introduction of routine culture investigations in chronic TB cases is likely to be highly cost-effective, due to the potential savings in terms of allocation of human resources and second-line TB drugs. Results obtained through molecular tests were, in our study, highly consistent with those obtained through classical culture and DST techniques, and may present significant advantages in terms of biosafety levels required and time to diagnosis.

The role of NTMs in the aetiology of TB-like syndromes has not been clearly elucidated in sub-Saharan African countries: a recently published comparative study supports a significant contribution [12]. In our series, a considerable proportion of NTMs were identified among both HIV-infected and uninfected chronic TB cases. As a consequence of these findings, the National Tuberculosis Programme (Ouagadougou, Burkina Faso) started a process to develop national guidelines for NTM management. Finally, the significant proportion of patients harbouring fully susceptible, vital $M$. tuberculosis strains suggests that poor adherence should always be considered as a cause of unsuccessful outcome of TB treatment.

Our study has several limitations. Culture tests were performed overseas on frozen samples. However, phenotypical investigations were supported by molecular tests, for which the use of frozen samples is widely accepted. The large majority of the 12 cases with negative cultures harboured DNA of drugsusceptible $M$. tuberculosis, suggesting the presence of nonvital mycobacteria at the end of an appropriate TB treatment. Our sample size was small and, though targetting the totality of registered chronic TB cases during the study period, we were able to analyse only $75 \%$ of those cases. However, exclusions were unbiased and unlikely to significantly affect the overall findings.

In summary, our data strongly support the recommendation that microbiological investigations (culture-based or molecular) should always be performed before starting second-line TB regimens and that chronic $\mathrm{TB}$ cases should be a priority population for microbiological investigations in low- and intermediate-resource settings. Political commitment is central to achieve this target, not only in Burkina Faso, but also in European countries where the Stop TB strategy is not yet fully implemented.

G. Badoum*, N. Saleri" ${ }^{\#,}$, M.S. Dembélé ${ }^{\mp}$, M. Ouedraogo*,
G. Pinsi", K. Boncoungou*, V. Bonkoungou ${ }^{+}$, E. Birba
P. Miotto
P. G.B. Migliori**, D.M. Cirillo ${ }^{f}$ and A. Matteelli"
${ }^{*}$ Dept of Pulmonary Care, "Yalgado" National Hospital,
University of Ouagadougou, "National Tuberculosis Program-
me, Ministry of Health, "National Council to Fight AIDS and 
STIs, Ouagadougou, and `Dept of Pulmonary Care, "Sanou Souro" National Hospital, University of Bobo Dioulasso, Bobo Dioulasso, Burkina Faso. "Institute of Infectious and Tropical Diseases, Brescia University, Brescia, ${ }^{f}$ Emerging Bacterial Pathogens Unit, San Raffaele Scientific Institute, Milan, and **WHO Collaborating Centre for TB and Lung Diseases, Fondazione S. Maugeri, Care and Research Institute, Tradate, Italy.

Correspondence: A. Matteelli, Infectious and Tropical Diseases Institute, University of Brescia, Piazza Spedali Civili, 125100 Brescia, Italy. E-mail: matteelli@med.unibs.it

Support Statement: This study was performed in the framework of the agreement for technical support requested by the Burkina Faso National TB Programme to the University of Brescia, Italy and the TB Supranational Reference Laboratory (SRL) at the San Raffaele Institute, Milan, Italy. It was partially supported by Italian cooperation: WHO grant SRL-HSR, University of Brescia and Global Fund grant BUR 404-T.

Statement of Interest: None declared.

Acknowledgements: We thank K. Théophile and K. Mariam (both Dept of Pulmonary Care, "Yalgado" National Hospital, Ouagadougou, Burkina Faso).

\section{REFERENCES}

1 World Health Organisation (WHO). Multidrug and Extensively Drug-Resistant TB (M/XDR-TB) - 2010 Global Report on Surveillance and Response. WHO/HTM/TB/2010.3. Geneva, WHO, 2010.
2 World Health Organization (WHO). Global Tuberculosis Control 2008. Surveillance, Planning, Financing. Geneva, WHO, 2008.

3 World Health Organization (WHO). Guidelines for the Programmatic Management of Drug Resistant Tuberculosis. Geneva, WHO, 2008.

4 Raviglione MC, Uplekar MW. WHO's new Stop TB Strategy. Lancet 2006; 367: 952-955.

5 World Health Organisation (WHO). Treatment of Tuberculosis: Guidelines for National Programmes, 3rd Edn. WHO/CDS/TB/ 2003.313. Geneva, WHO, 2003.

6 National Tuberculosis Program (NTP), Ministry of Health Burkina Faso: Guide technique de lutte contre la tuberculose 2008 [Technical TB guide 2008. Edited by the National Tuberculosis Programme]. Ouagadougou, NTP, 2008.

7 Rusch-Gerdes S, Pfyffer GE, Casal M, et al. Multicentre laboratory validation of the Bactec MGIT 960 technique for testing susceptibilities of Mycobacterium tuberculosis to classical second line drugs and newer antmicrobials. J Clin Microbiol 2006; 44: 688-692.

8 Miotto P, Saleri N, Dembelé M, et al. Molecular detection of rifampin and isoniazid resistance to guide chronic TB patient management in Burkina Faso. BMC Infect Dis 2009; 9: 142.

9 Chen-Yuan C, Enarson DA, Fujiwara PI, et al. Strategies of extensively drug resistance TB risk management for health workers and other care givers. Expert Rev Respir Med 2008; 2: 47-54.

10 Sotgiu G, Ferrara G, Matteelli A, et al. B. Epidemiology and clinical management of XDR-TB: a systematic review by TBNET. Eur Respir J 2009; 33: 871-881.

11 Migliori GB, Besozzi G, Girardi E, et al. Clinical and operational value of the extensively drug-resistant tuberculosis definition. Eur Respir J 2007; 30: 623-626.

12 Buijtels PCAM, van der Sande MAB, de Graaff CS, et al. Nontuberculous mycobacteria, Zambia. Emerg Infect Dis 2009; 15: 242-248.

\section{Impaired pulmonary function and the risk of tuberculosis: a population-based cohort study}

\section{To the Editors:}

It is well known that pulmonary tuberculosis (TB) can cause lung impairment leading to chronic obstructive pulmonary disease (COPD) [1], but less is known to what extent impaired lung function increases the risk of TB. A case-control study from the UK found patients with emphysema to have a threefold increased risk of $\mathrm{TB}$, adjusted for smoking and the use of corticosteroids [2]. A cohort study from Denmark found moderate-to-severe COPD to be associated with a two- to three-fold increased risk of hospitalisation with TB [3]. We have previously shown that patients hospitalised with COPD have a three-fold increased risk of active TB compared with population controls [4]. In the present study, we examine the risk of active TB in relation to lung function in a cohort who underwent spirometry in 1974-1992.

Between 1974 and 1992, 22,444 males and 10,902 females, born 1921-1949, participated in a health screening programme, the Malmö Preventive Project. Complete birth cohorts, born in pre-specified years, from the city of Malmö were invited; the overall participation proportion was $\sim 70 \%$ [5]. The screening included physical examination, spirometry, blood samples and assessment of lifestyle factors by means of a self-administered questionnaire. Some questions varied between the cohorts. We excluded 4,413 individuals because of missing spirometry data; 26 individuals were excluded because of missing data on either body mass index (BMI), smoking or immigration status, leaving a total of 28,907 individuals in final analysis $(21,174$ males and 7,733 females).

BMI as was calculated as mass $/$ height $^{2}\left(\right.$ in $\mathrm{kg} \cdot \mathrm{m}^{-2}$ ). Individuals were regarded as having diabetes mellitus if they answered positively to the question "Do you have diabetes mellitus?" or their fasting blood glucose level was $\geqslant 6.1 \mathrm{mmol} \cdot \mathrm{L}^{-1}$.

Around $96 \%$ of the cohort $(n=27,789)$ answered questions on alcohol. Individuals were regarded as having "problematic drinking" if they answered affirmatively to four or more questions on drinking habits and were regarded as having 